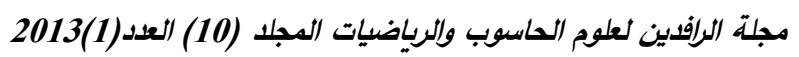

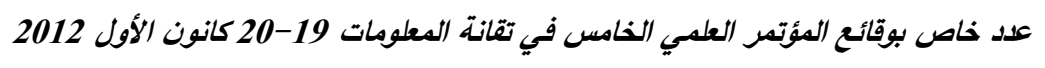

\section{Representation of the Wavelet Transform 2D using A 5/3 Filter on the Field Programmable Gate Array}

Maha Abdul Rahman Hasso

College of Computer Science and Mathematics

University of Mosul, Mosul, Iraq

Received on: 16/10/2012

Accepted on: 30/01/2013

\begin{abstract}
Recently the Wavelet Transform has gained a lot of popularity in the field of signal and image processing, this is due to its capability of providing both time and frequency information simultaneously. In this paper, focus on used Discrete Wavelet Transform 2-dimenssion (2D- DWT) based on conventional approach, convolution, to image processing and implementation by using FPGA (Field Programmable Gate Array), due to many researches were implemented on this Hardware in recent years ,using VHDL.

In this paper, has been proposed two VHDL architectures to implementation the conventional of the Daubechies 5/3-tap biorthogonal filter bank, a simple straightforward one and an optimized one, substituting the multipliers used for scaling with shift - add operations. The architecture of optimized approaches were designed and implemented on FPGA, type of Xilinx XC3S500E Spartan-3E.
\end{abstract}

Keywords: Discrete Wavelet Transform 2D, convention, Field Programmingable Gate Array.

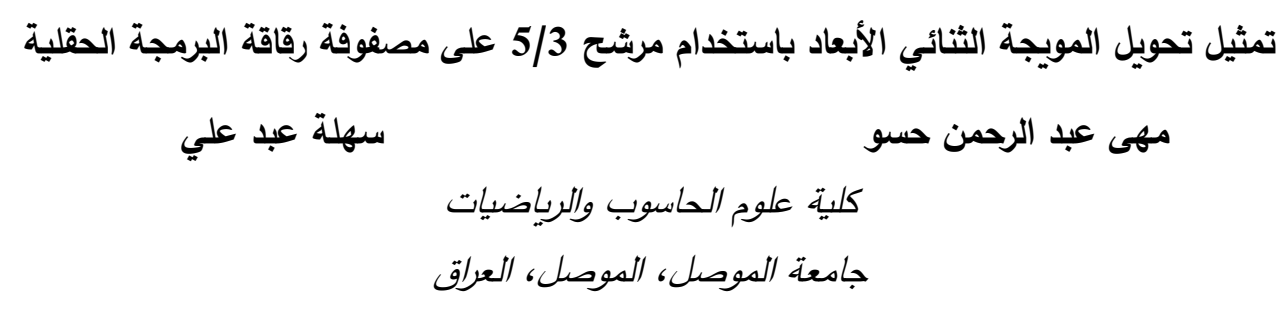

تاريخ قبول البحث: 2013/01/30

تاريخ استلام البحث: 2012/10/16

الملخص

كسبت تحويلات المويجة (DWT) مؤخرا الكثير من الثعبية في حقل الإشارة ومعالجة الصورة، وهذا

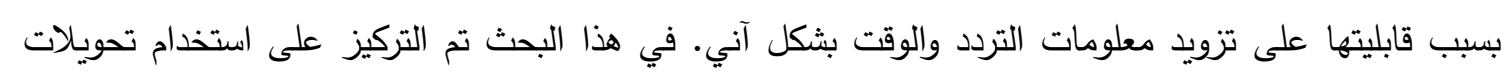
المويجة ذات البعدين (2D- DWT) باعتماد الطريقة التقليدية، الالتفاف الرياضي (convolution)، لمعالجة الصورة وتطبيقه باستخدام جهاز Field Programmable Gate Array (FPGA) إذ أن الكثير من البحوث تم تطبيقها على هذه الأجهز المادية وبشكل ملحوظ في السنوات الأخيرة للاستفادة من محاسنها، باستخدام لغة

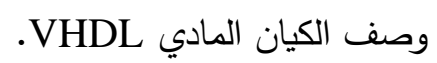


تم في هذا البحث اقتراح معماريتين لـ VHDLechies 5/3 - لتطبيق الالتفاف الرياضي للمرشح tap biorthogonal filter bank

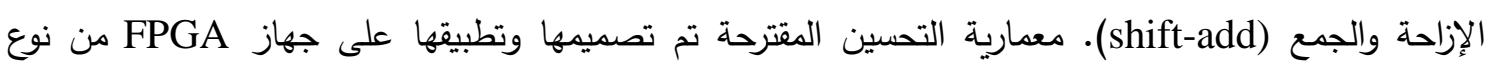
.Xilinx XC3S500E Spartan-3E الكلمات المفتاحية: تحويل المويجة الثنائي الأبعاد المنفصلة، الالتفاف الرياضي، مصفوفة رقاقة البرمجة الحقلية. 1

تحويلات المويجة المنفصلة Discrete Wavelet Transform (DWT) للإثارة هي بديل عن تمثيل الوقت-التردد الموجود مثل تحوير فورير المتقطع وتحوير الجيب تمام، ولقد أصبح شائعاً في العديد من تطبيقات

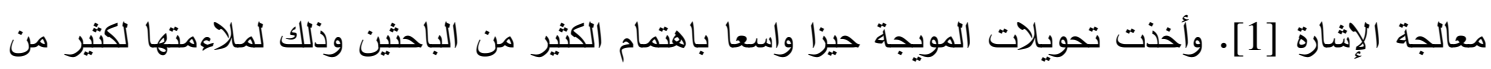

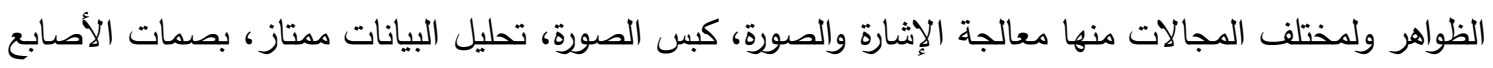
الرقمية، تحسين الصورة، تقليل الضوضاء عن الإثارات وإزالتها، وتحليل إثارات الزلازل، الخ[2]. إذ ظهرت المعلومات والتحديدات داخل الصور في الترددات العالية بينما توجد المعلومات ذات الخاصية البعيدة عن تحديدات الحافات (smoothing في الترددات الواطئة للصورة. لمعالجة الأوساط المتعددة، تم اعتماد تحويل المويجة لكبس الصورة بسبب أدائها الأفضل من تحوير جيب لكودي التمام المنفصل التقليدي لكبس الصورة، خصوصا لتطبيقات نسبة البت المنخفضة. لذا الكثير من أجهزة

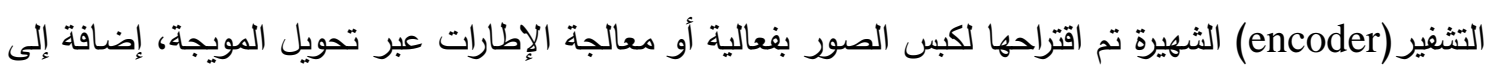
ذلك، هذا التحويل مستخدم في معيار 2000 JPEG، معيار جديد لكبس الصورة، واعتماد مرشحي المويجة 9/7 و5/3 بوصفها مرشحات افتراضية للكبس بفقدان ودون فقدان على التوالي. إن تحويل المويجة يطبق أما باعتماد

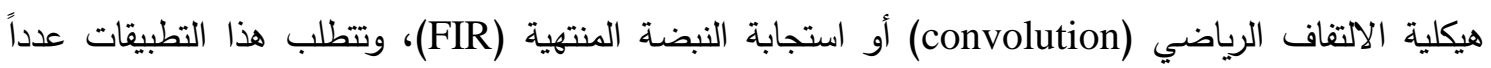
كبيراً من العمليات الحسابية ومساحة خزن، أو اعتماد هيكلية مخطط الرفع (lifting scheme) للمويجة والذي الزيانية اقترح من قبل Swelden. تم اقتراح العديد من المعماريات لتحويل المويجة في السنوات السابقة[3]، ففي المعمارية المقترحة في[4] دمجت التحويل بفقدان ودون فقدان، وفي [5] المعمارية المقترحة خفضت بثكل ملحوظ عدد فئ الضوارب (multiplier)، الجوامع (adder) والسجلات إضافة إلى كمية الوصول للذاكرة الخارجية وأدت إلى تقليل كلفة الجهاز واستهلاك القدرة بشكل كفوء في التصميم.

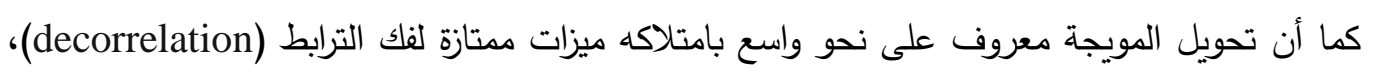

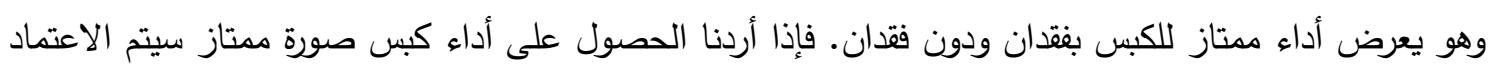
على هذا التحويل لتحقيقه شرط إعادة بناء مثالي (perfect reconstruction PR)، لذا هذا التحويل مستخدم في

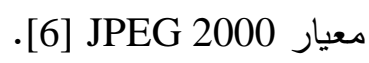
بسبب تزايد عدد التطبيقات في مناطق مختلفة، أصبح من الضروري استخدام التطبيق المادي لتحويل المويجة المنفصل لكفاءتها. في أي تصميم كفوء يجب أن يؤخذ في الحسبان عدد من السمات مثل المنطقة، استهلاك كهربائي، طاقة إنتاجية، الخ.تقنيات مثل الـ pipelining, distributed arithmetic تساعد في تحقيق هذه الطلبات. في الكثير من تطبيقات الصوت، الصورة، الفيديو، التسجيل الصوتي، أكثر المشاكل هي خزن الذاكرة ونقل البيانات، لذا عند التصميم يجب أن تؤخذ هذه العوامل بنظر الاعتبار[7]. في هذا البحث تمت برمجة 
تحويلات المويجة المنفصلة ذات بعدين (2D-DWT) باستخدام ماتلاب وكذلك باستخدام لغة وصف الكيان المادي VHDL لإمكانية تطبيق الـ 2D-DWT مادياً على رقاقة الـ FPGA.FPGA يمتلك خصائص دوائر التطبيق المتكاملة application specific integrated circuits) ASICs مع محاسن إمكانية إعادة التهيئة، إذ يحتوي على مصفوفة من الخلايا المنطقية وقنوات توجيه تدعى روابط (interconnects) التي يمكن برمجتها

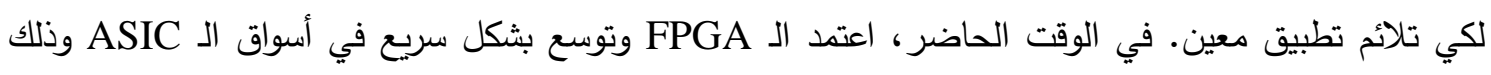

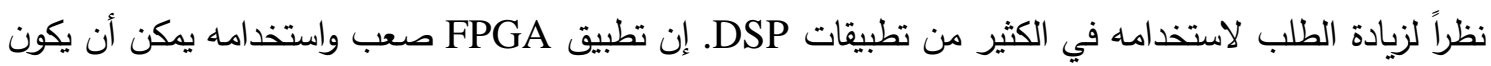
تحديا للقابليات الحسابية الغير جيدة عند مقارنته مع معالجات DSP ذات الغرض العام. لعلى على أية حال، الفائدة

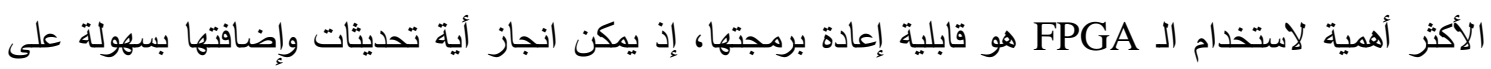
عكس ما موجود في حالة الـ ASICs التقليدية.

\section{The Wavelet Transform تحويل المويجة}

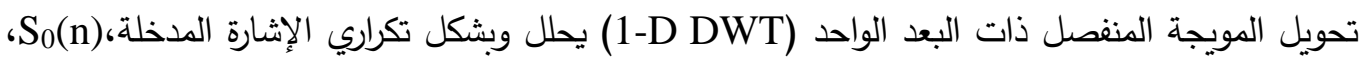
إلى معاملي التقريب والتقصيل.لنفرض أن Si(n) و Wo يمثلان التقريب والتفصيل، على التوالي، للإثشارة عند مستوى التحليل i. معامل التقريب للإثارة عند المستوى i+1 يحسب باستخدام المعادلة الآتية: $S_{i+1}(n)=\sum_{k=0}^{L-1} h(k) S_{i}(2 n-k)$,

ومعامل التفصيل للإثشارة عند المستوى 1+1 يحسب باستخدام المعادلة الآتية:

$W_{i+1}(n)=\sum_{k=0}^{L-1} g(k) S_{i}(2 n-k)$.

إن المعادلات (1) و(2) تصف العمليات الحسابية لتحويل المويجة (DWT) المنفصل.هذه التقنية

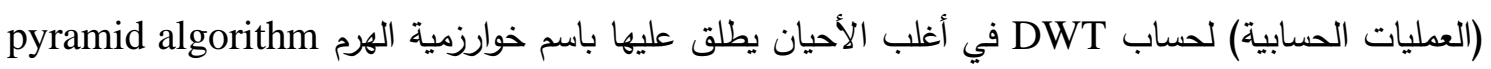
أو خوارزمية Mallat's. الإثارة المدخلة يمكن إعادة تركيبها (استرجاعها) من معاملات المويجة نفسها باستخدام معادلة معكوس تحويل المويجة المنفصل (IDWT):

$S_{i-1}(n)=\sum_{k}\left\{S_{i}(k) h^{\prime}(n-2 k)+W_{i}(k) g^{\prime}(n-2 k)\right\}$.

إن تحويل المويجة ذات بعدين (2D DWT and IDWT) يمكن تطبيقه باستخدام معمارية المرشح

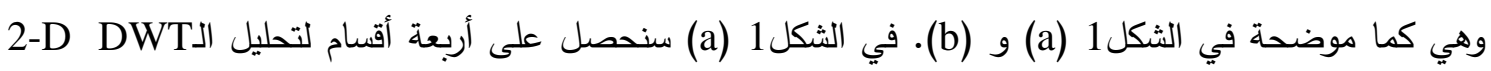

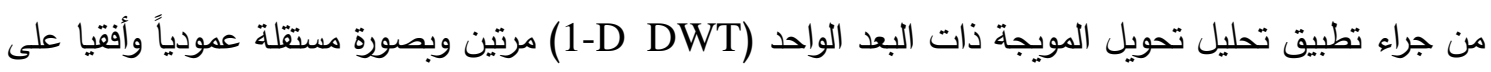
الصورة. وبالطريقة نفسها بالنسبة لـ 2-D IDWT لإعادة تركيب الصورة واسترجاعها عن طريق تطبيق الـ بصورة عمودية وأخرى أفقية. لأجل التطبيق سنفرض أن: (i) حجم الصورة هو N x NT مستويات التحليل للصورة هو J و (iii) مرشحات الإمرار الواطئ والعالي هي مرشحات متمائلة ذات حجم K2 

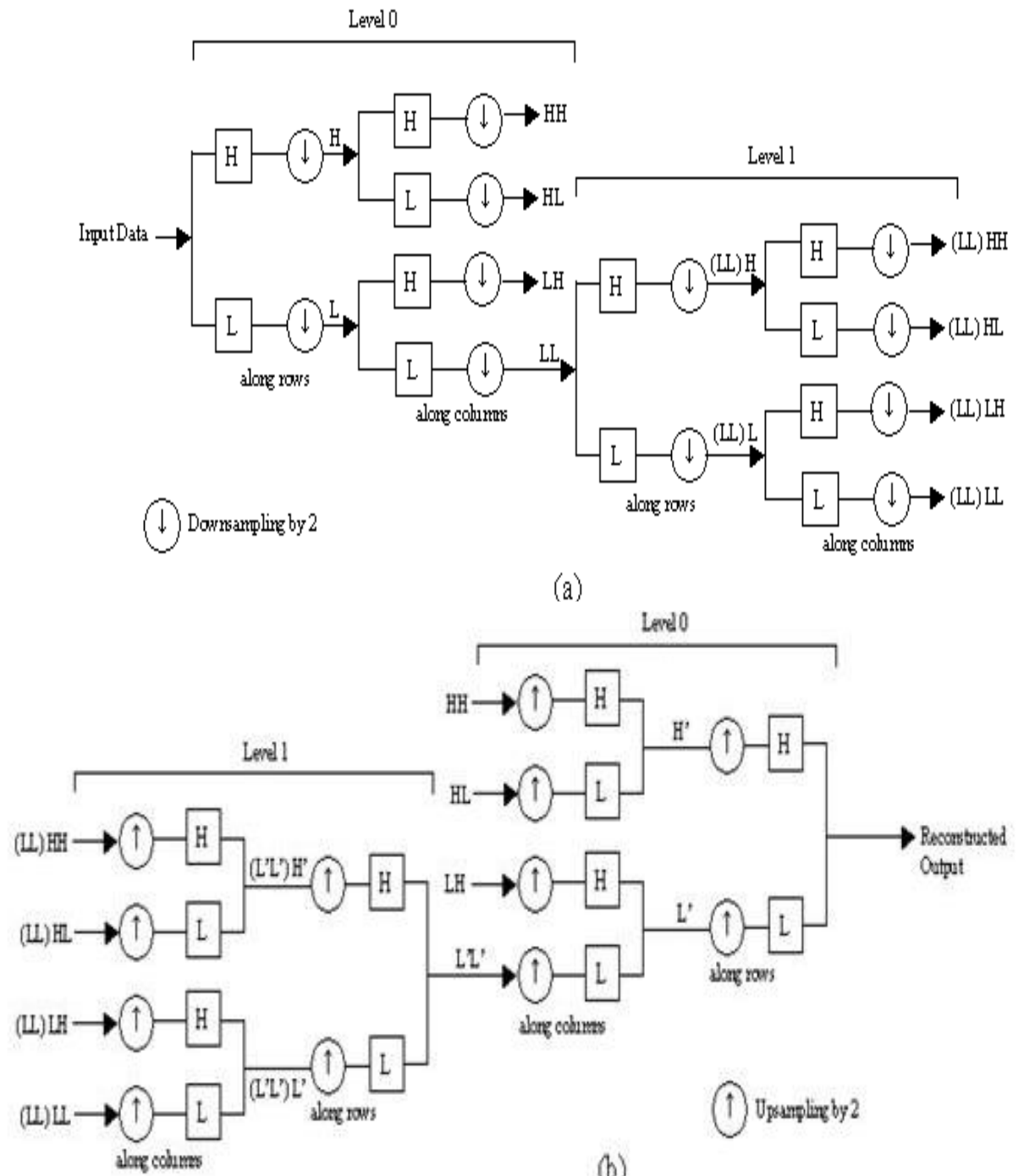

(b)

2-D IDWT (b) 2-D DWT(a) 2=J الثكل (1). هيكل المرشح عند

في معمارية مرشح الـ 2-D DWT، إخراجات مرشحي الإمرار الواطئ والعالي بعد (a+1) مرحلة حسابية على طول الصف ترمز بـ (LL) هي إخراجات مرشحي الإمرار العالي والواطئ بعد مراحل من

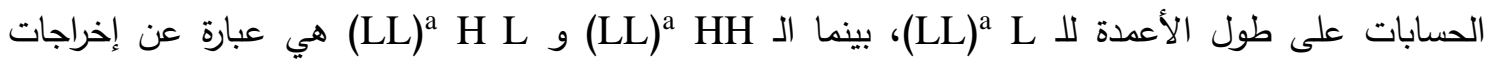

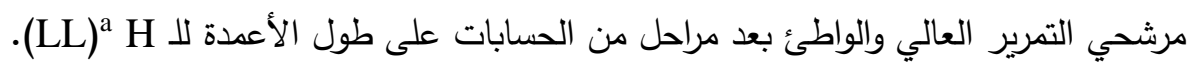

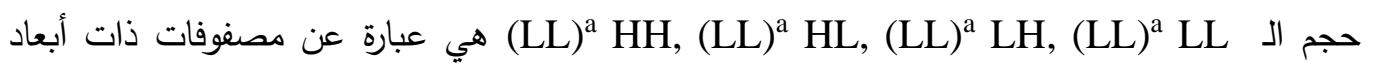

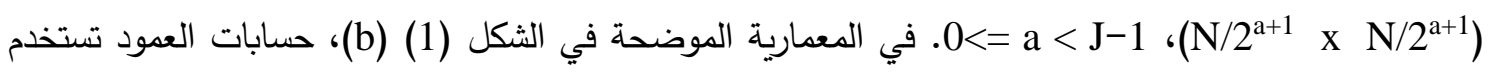

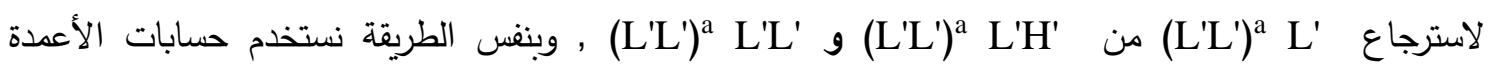

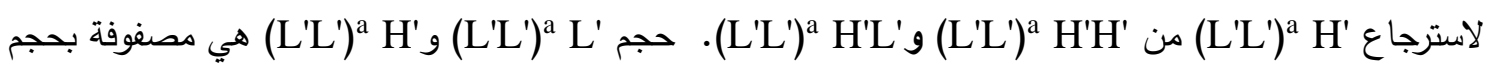

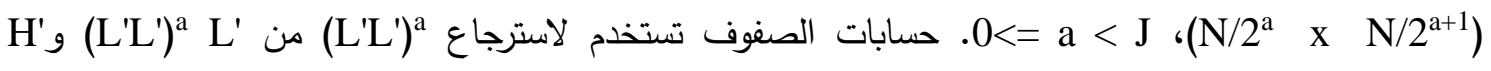

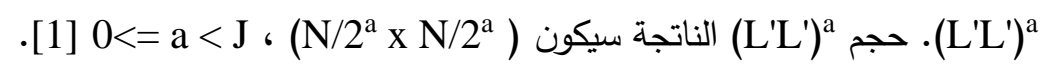


إن العدد الكلي لحسابات مرشحات التحليل لصورة ذات حجم N x N بعد المستوى m لل 2-D DWT يمكن حسابه من المعادلة التالية:

$$
N^{2}+\left(\frac{1}{4}\right) N^{2}+\left(\frac{1}{4}\right)^{2} N^{2}+\cdots+\left(\frac{1}{4}\right)^{m-1} N^{2}=\frac{4}{3}\left(1-4^{-m}\right) N^{2}
$$

طبقاً للمعادلة السابقة (4) سيكون الحد الأعلى لعدد حسابات مرشحي الإمرار الواطئ والعالي هو 4/3 [8]. 3. Convolution Filters مرشحات الاتفاف الرياضي

تم استخدام الطريقة التقليدية لحساب تحويلات المويجة وهي طريقة الالتفاف الرياضي باستخدام 5/3-tap biorthogonal filter

\section{Forward filters}

$$
\begin{aligned}
& Y_{2 n}=\operatorname{cl} 0 * X_{2 n}+ \\
& \text { cll } *\left(X_{2 n-1}+X_{2 n+1}\right)+\quad \operatorname{chl} *\left(X_{2 n}+X_{2 n+2}\right) \\
& c l 2 *\left(X_{2 n-2}+X_{2 n+2}\right)
\end{aligned}
$$

$$
\text { معاملات المرشحين: }
$$

\section{Inverse filters}

$$
\begin{aligned}
& X_{2 n}=c l O * Y_{2 n}+ \\
& \text { cll* }\left(Y_{2 n-1}+Y_{2 n+1}\right) \\
& \mathrm{clO}=1 \\
& c l 1=0.5 \\
& X_{2 n+1}=\operatorname{ch} 0 * Y_{2 n+1}+ \\
& \text { chl } *\left(Y_{2 n}+Y_{2 n+2}\right)+ \\
& \operatorname{ch} 2 *\left(Y_{2 n-1}+Y_{2 n+3}\right) \\
& \text { chO }=0.75 \\
& \text { ch } 1=-0.25 \\
& \text { ch } 2=-0.125
\end{aligned}
$$

إن تصميم الالتفاف الرياضي باستخدام لغة وصف الكيان المادي (VHDL) مسالة صعبة جداً وذلك بسبب استخدام حسابات رياضية معقدة مثل الجامع، الضارب والقاسم (adders, multipliers, and dividers) لحساب الإخراج.

إن استخدام العمليات الحسابية (الرياضيات) في الـ FPGA يؤدي إلى بطئ الأداء. الكثير من المصمدين يفضلون التقنيات التي تقلل من اعتمادية التصميم على الحسابات الرياضية المعقدة، لكن مازالت تستخدم

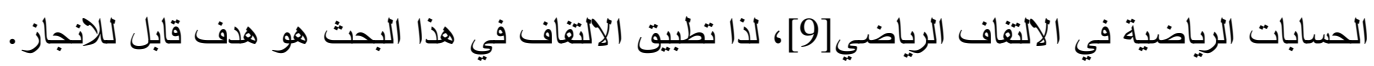

\section{The 5/3-tap Filters Implementation تطبيق المرشح}

$$
\text { Multiplier - based باعتماد الضارب (i) }
$$

إن المعمارية لتطبيق المرشح باستخدام الضارب موضحة في الثكل(2) وهي معمارية بسيطة. في هذا التطبيق سيتم الحاجة إلى خمسة ضوارب (multipliers) وستة جوامع (adders). 


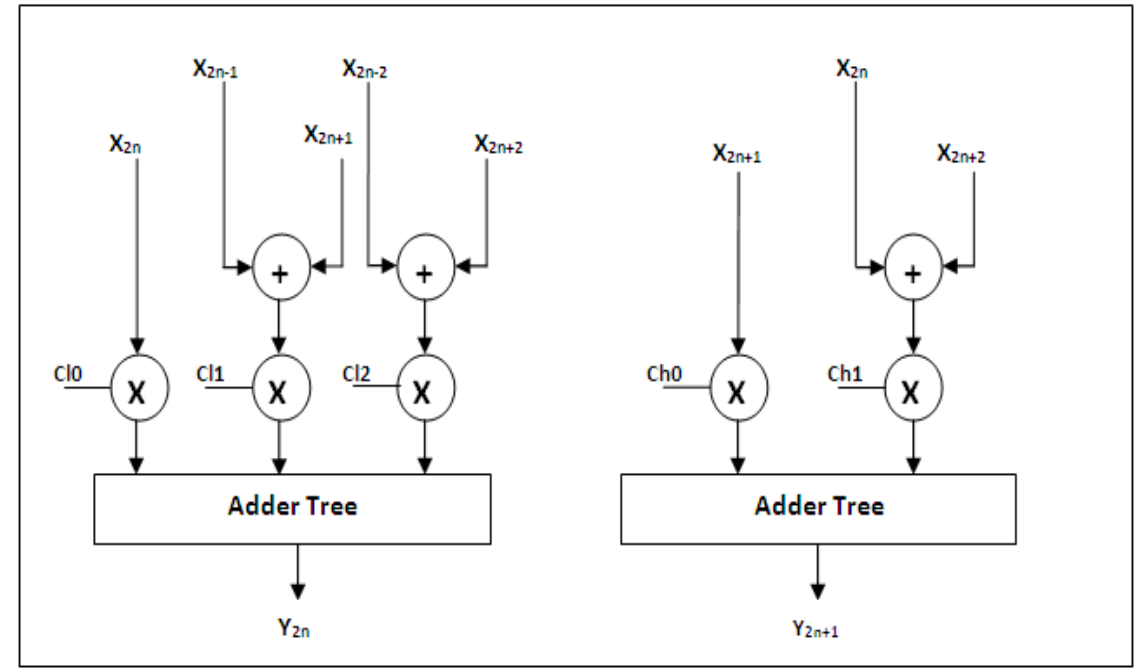

الشكل (2). معمارية المرشحات بطريقة الالتفاف الرياضي باعتماد الضارب

Optimized, adder - based باعتماد الجامع (ii)

يمكن تطبيق المرشح باستبدال الـ multipliers بعمليات الإزاحة والجمح، وهذا يؤدي إلى تطبيق مضغوط أكثر وأسرع مقارنةً مع التطبيق السابق الذي استخدم الضارب (multipliers). الرسم التخطيطي لمرشحي الإمرار الواطئ والعالي موضح في الثكل(3):

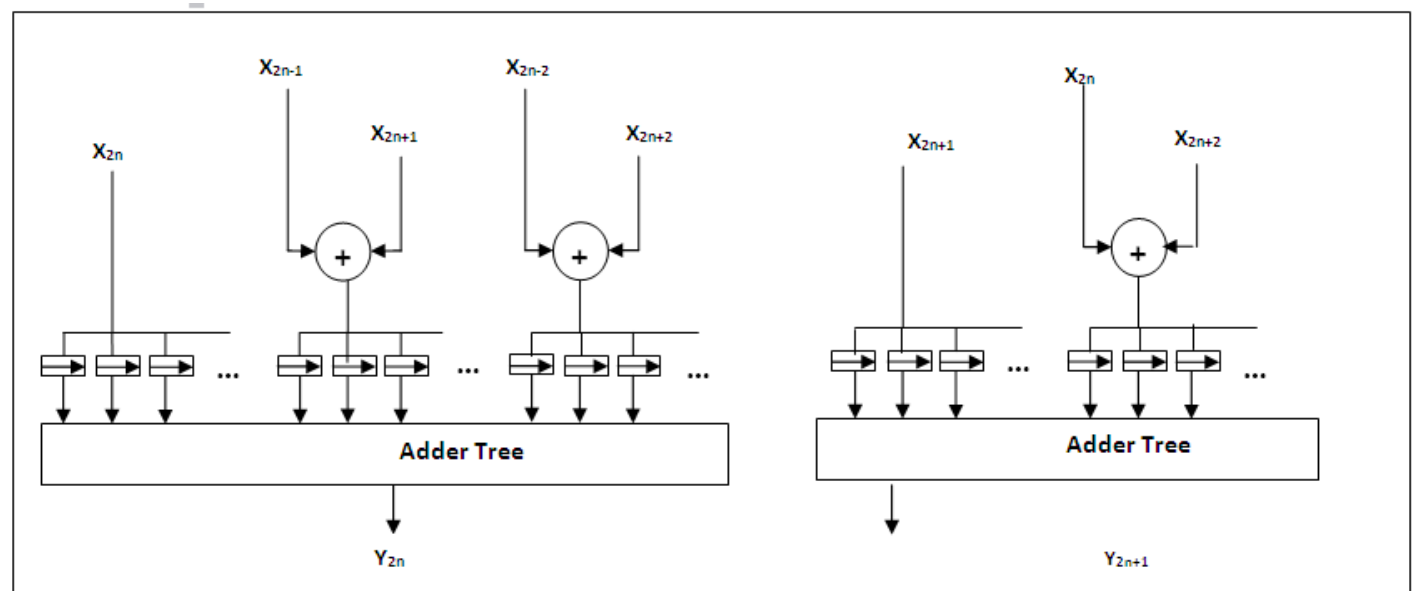

الثكل (3). معمارية المرشحات بطريقة الالتفاف الرياضي باعتماد الجامع

5. تطبيق خوارزمية تحويل المويجة

\section{Implementation of DWT and IDWT algorithm}

db 5/3-tap تمبيق الخوارزمية في ماتلاب باستخدام طريقة ضرب المصفوفات باستخدام مرشح biorthogonal 


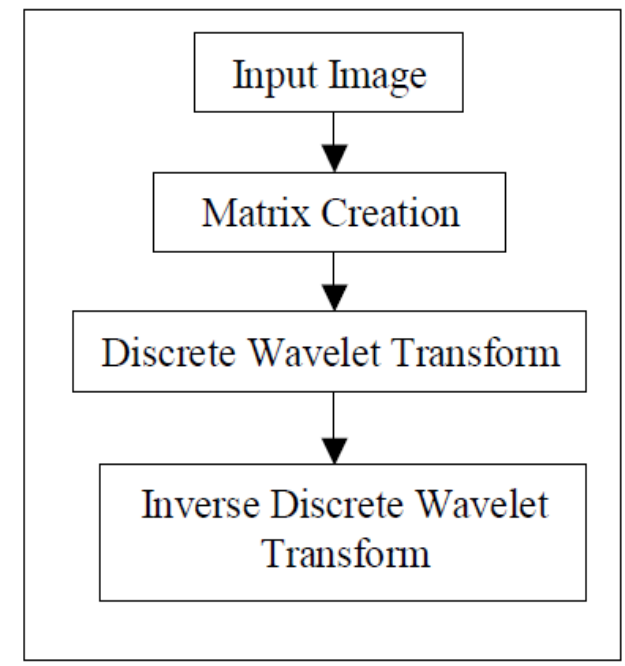

الثكل (4). خطوات تطبيق الخوارزمية في ماتلاب.

ثم تم بعدها تطبيق الخوارزمية باعتماد معمارية التحسين لتطبيق الالتفاف الرياضي لتحويلات المويجة باستخدام

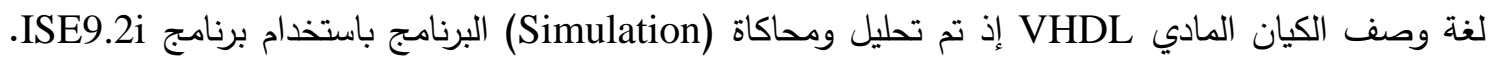
الثكل (5) يوضح المعمارية لخوارزمية تحويل المويجة ذات بعدين لمستوى تحليل واحد للصورة، والثكل (6) تونة يوضح نتائج Simulation التي تم الحصول عليها من تطبيق هذه المعمارية لمستوى واحد.

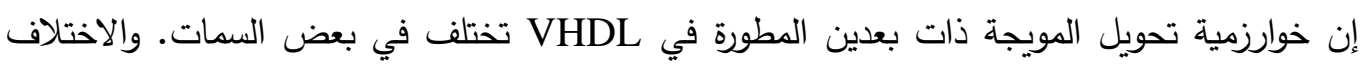

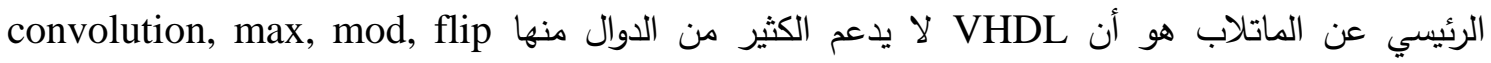

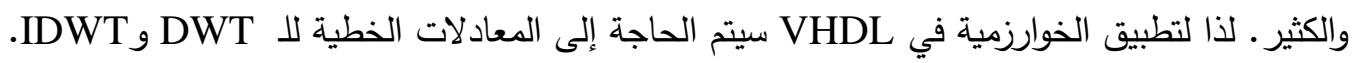

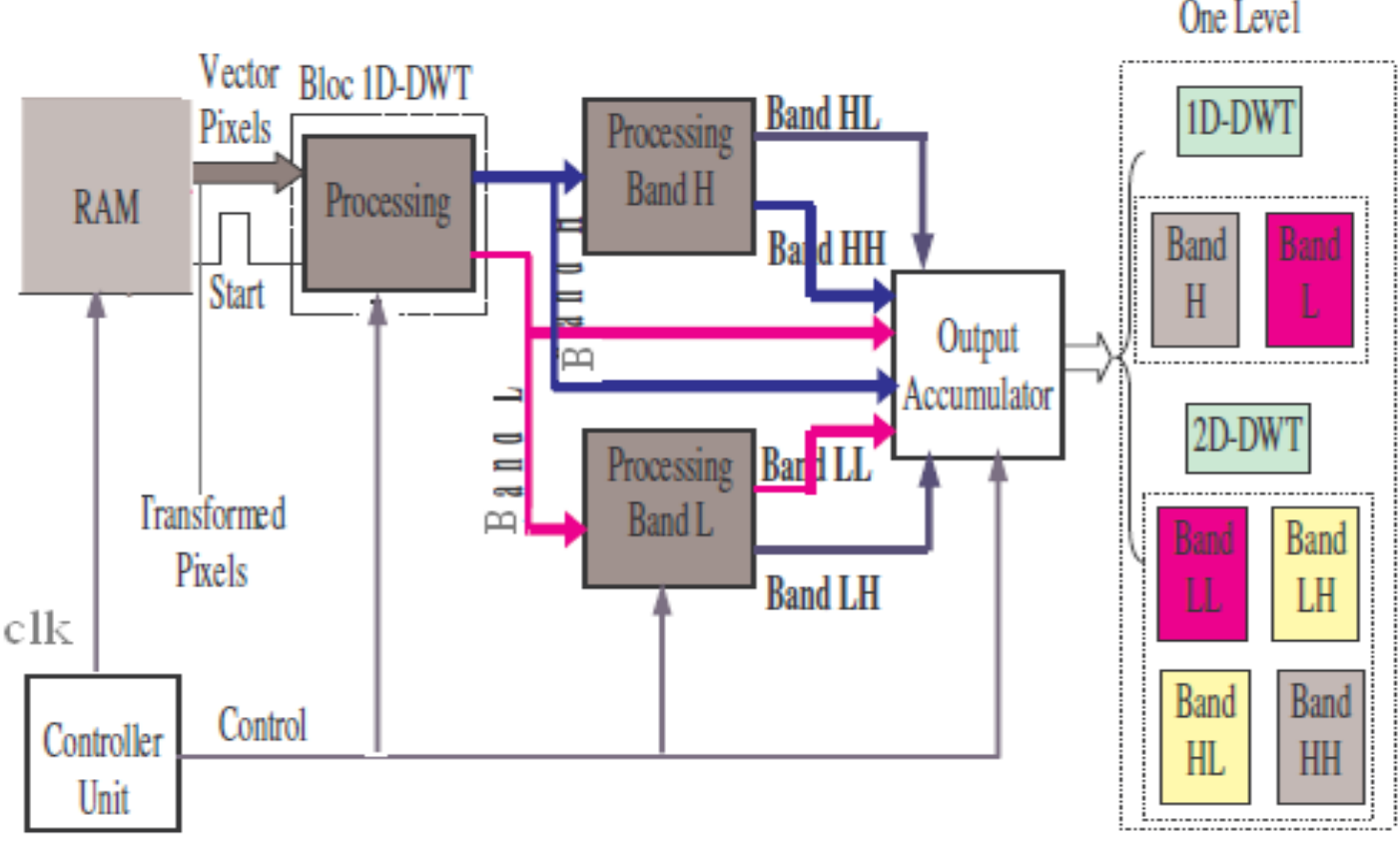

الثكل (5). التصميم لمعمارية تحويل المويجة المنفصل في VHDL 


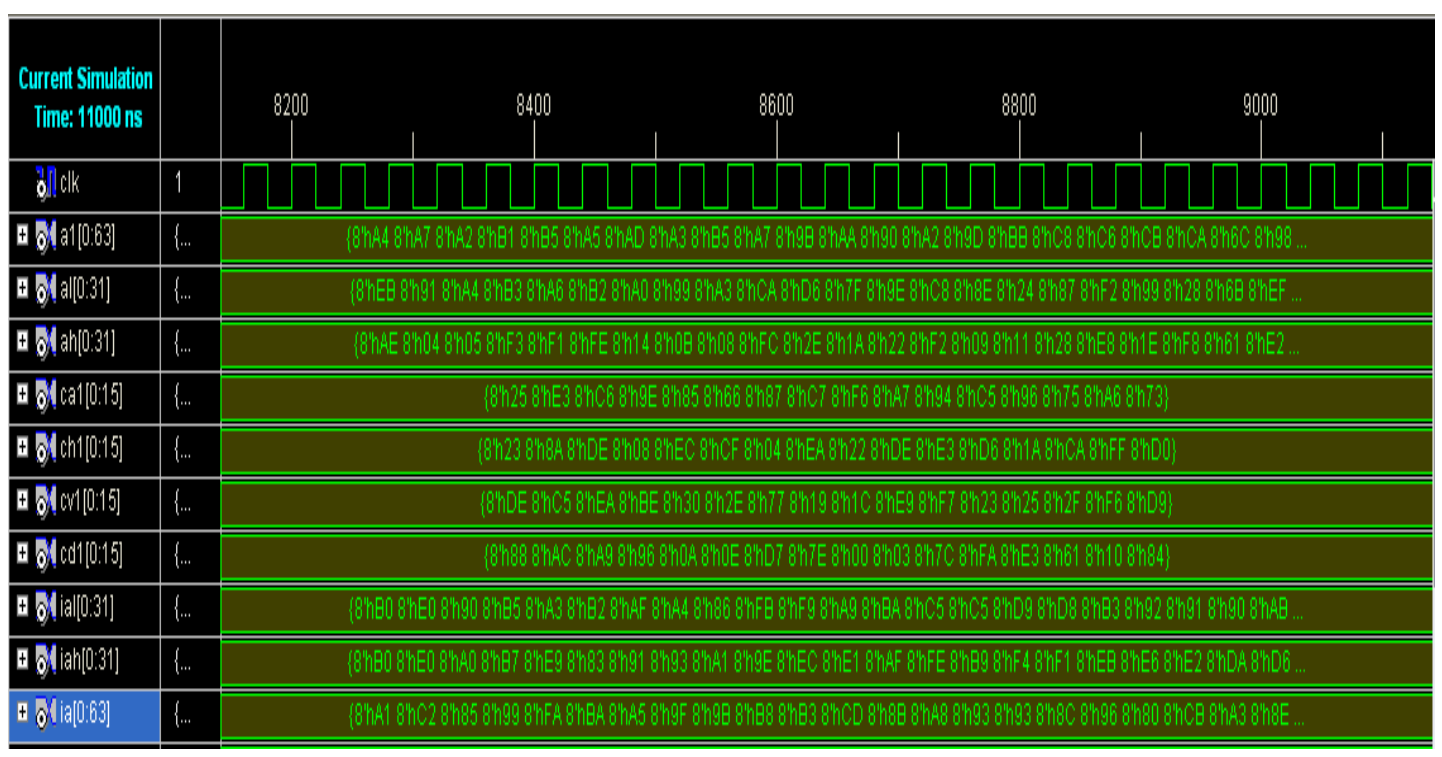

الثكل (6imulation) (6) نتائج محاكاة وصف الكيان المادي لخوارزمية تحويل المويجة المتقطعة ذات

بعدين

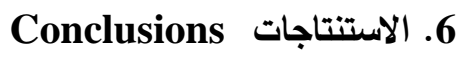

تم في هذا البحث استخدام خوارزمية تحويل المويجة المنفصل وتطويرها واختبارها في ماتلاب. ثم تحديث

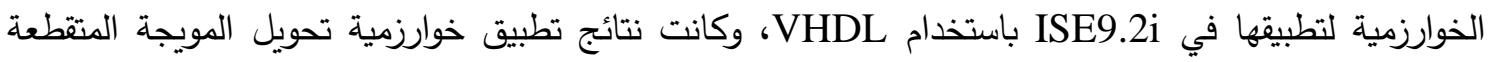
ذات بعدين باعتماد معمارية الجامع والإزاحة المقترحة في هذا البحث بدل عملية الضرب (اي معمارية الضارب) لتطبيق المرشح 5/3 موضحة في الجدول التالي، هذه النتائج توضح تطبيقات Xilinx FPGA لهذه المعمارية

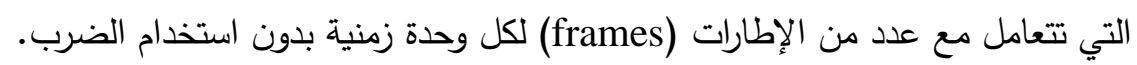

كم أن من نتائج المحاكاة Simulation الموضحة سابقاً يمكن أن نستتتج أن الخوارزمية تعمل بشكل الفئل صحيح ، وان كود VHDL وُلد (synthesized) لتحقيق المعمارية إلى المستوى المطلوب والتي تكون جاهزة للتطبيق على الجهاز •

الجدول 1: نتائج تطبيقات FPGA للمعمارية المقترحة.

\begin{tabular}{|l||llll||}
\hline Number of Slices & 1195 & out of 4656 & $25 \%$ \\
\hline \hline Number of Slice Flip Flops & 1202 & out of 9312 & $12 \%$ \\
\hline \hline Number of 4 input LUTs & 2223 & out of 9312 & $23 \%$ \\
No. used as logic & $\mathbf{1 7 8 7}$ & & & \\
No. used as Shift registers & $\mathbf{2 4 4}$ & & & \\
No. used as RAMs & $\mathbf{1 9 2}$ & & & \\
\hline Number of bonded IOBs & 15 & out of 232 & $6 \%$ \\
\hline Number of GCLKs & 1 & out of 24 & $4 \%$ \\
\hline \hline
\end{tabular}




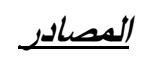

[1] Kavish Seth, S.Srinivasan, "VLSI Implementation of 2-D DWT/IDWT Cores using 9/7-tap filter banks based on the Non-expansive Symmetric Extension Scheme", Department of Electrical Engineering, IEEE, 2002.

[2] Gaurav Tewari, Santu Sardar, K. A. Babu, "High-Speed \& Memory Efficient 2D DWT on Xilinx Spartan3A DSP using scalable Polyphase Structure with DA for JPEG2000 Standard", IEEE, 2011.

[3] Dhaha Dia, Medien Zeghid, Taoufik Saidani, Mohamed Atri, Belgacem Bouallegue, Mohsen Machhout and Rached Tourki, "Multi-level Discrete Wavelet Transform Architecture Design", Proceedings of the World Congress on Engineering, Vol. I, WCE 2009, July 1 - 3, London, U.K., 2009.

[4] M. Martina and G. Masera, "Multiplierless, Folded 9/7-5/3 Wavelet VLSI Architecture", IEEE Transactions on Circuits and Systems- II: Express Briefs, vol.54 No. 9, September 2007.

[5] X. Chengyi, T. Jinwen and L. Jian, "Low complexity reconfigurable architecture for the 5/3 and 9/7 discrete wavelet transform", Journal of Systems Engineering and Electronics vol. 17 No.2, pp. 303-308, 2006.

[6] Guoan Yang \& Huub Van de Wetering \& Songjun Zhang, "Optimization Design of Biorthogonal Wavelet Filter Banks for Extending JPEG 2000 Standard Part2", J Sign Process Syst (2012).

[7] D. U. Shah1, C. H. Vithlani2, "Efficient Implementation of Discrete Wavelet Transforms Using FPGAS", International Journal of Advances in Engineering \& Technology, Sept 2011.

[8] Chu Yu and Sao-Jie Chen, "Design of an Efficient Vlsi Architecture for 2-D Discrete Wavelet Transforms", IEEE, November 30, 1999.

[9] Anthony Edward Nelson, "Implementation of Image Processing Algorithms on FPGA Hardware", M.Sc. Thesis, Electrical Engineering of Vanderbilt University, May 2000. 